PROCEEDINGS OF THE

AMERICAN MATHEMATICAL SOCIETY

Volume 128 , Number 12, Pages 3513-3517

S 0002-9939(00)05463-0

Article electronically published on May 18, 2000

\title{
A SHARP SCHWARZ INEQUALITY ON THE BOUNDARY
}

\author{
ROBERT OSSERMAN
}

(Communicated by Albert Baernstein II)

\begin{abstract}
A number of classical results reflect the fact that if a holomorphic function maps the unit disk into itself, taking the origin into the origin, and if some boundary point $b$ maps to the boundary, then the map is a magnification at $b$. We prove a sharp quantitative version of this result which also sharpens a classical result of Loewner.
\end{abstract}

A recent paper of the author and Min $\mathrm{Ru}[\mathrm{OR}$ makes use of a new Schwarztype lemma on surfaces (Lemma 2.1 of that paper.) An effort to understand the geometry underlying the proof of that lemma led the author to a much more general Ahlfors-Schwarz Lemma on surfaces, and to an apparently new link between lemmas of that sort and the standard comparison theorems of Riemannian geometry [.

Looking back at the comparable situation in the classical case, one finds the following:

The standard Schwarz Lemma states that an analytic function $f(z)$ mapping the unit disk into itself, with $f(0)=0$, must map each smaller disk $|z|<r<1$ into itself and (as a result) satisfy $\left|f^{\prime}(0)\right| \leq 1$. Furthermore, unless $f$ is a rotation, one has strict inequality $\left|f^{\prime}(0)\right|<1$ and $f$ maps each disk $|z| \leq r<1$ into a strictly smaller one.

It is an elementary consequence of Schwarz' Lemma that if $f$ extends continuously to some boundary point $b$ with $|b|=1$, and if $|f(b)|=1$ and $f^{\prime}(b)$ exists, then $\left|f^{\prime}(b)\right| \geq 1$. It is also true that one again has strict inequality unless $f$ is a rotation, but that does not follow from the standard Schwarz inequality; one needs a stronger form where one has a quantitative bound on how much each disk $|z| \leq r<1$ is shrunk if $f$ is not a rotation.

These facts are well known. (See, for example, Carathéodory [C2], §§296-301.) However, what does not seem to have been observed, at least in print, is that there is a sharp boundary inequality (Lemma 1 below) from which the above facts follow. Although the components of the proof can all be found in the literature 1 (in particular, the sections of Carathéodory's book cited above), it seems worth presenting the sharp inequalities of Lemmas 1 and 3 below together with direct elementary proofs.

Received by the editors June 15, 1998 and, in revised form, January 26, 1999.

2000 Mathematics Subject Classification. Primary 30C80.

Key words and phrases. Schwarz Lemma.

The author's research at MSRI is supported in part by NSF grant DMS-9701755.

${ }^{1}$ I would like to thank the referee for providing details of this, and for other useful comments.

(C)2000 American Mathematical Society 
Lemma 1 (The boundary Schwarz Lemma). Let $f(z)$ satisfy

(a) $f(z)$ is analytic for $|z|<1$,

(b) $|f(z)|<1$ for $|z|<1$,

(c) $f(0)=0$,

(d) for some $b$ with $|b|=1, f(z)$ extends continuously to $b,|f(b)|=1$, and $f^{\prime}(b)$ exists.

Then

$$
\left|f^{\prime}(b)\right| \geq \frac{2}{1+\left|f^{\prime}(0)\right|} .
$$

Corollary 1. Under hypotheses (a) - (d),

$$
\left|f^{\prime}(b)\right| \geq 1
$$

and

$$
\left|f^{\prime}(b)\right|>1 \text { unless } f(z)=e^{i \alpha} z, \alpha \text { real. }
$$

Proof. Inequalities (2) and (3) follow immediately from (1) together with the standard Schwarz Lemma.

Corollary 2. Let $f$ satisfy conditions (a), (b), (c) of the lemma, and suppose that $f$ extends continuously to an arc $C$ on $|z|=1$, with $|f(z)|=1$ on $C$. Then the length $s$ of $C$ and the length $\sigma$ of $f(C)$ satisfy

$$
\sigma \geq \frac{2}{1+\left|f^{\prime}(0)\right|} s .
$$

Proof. By the reflection principle, $f$ extends to be analytic on the interior of $C$ and therefore satisfies condition (d) of Lemma 11. Hence (4) follows from (1).

Remarks 1. 1. Again by the standard Schwarz Lemma, (4) implies that $\sigma \geq s$, and $\sigma>s$ unless $f$ is a rotation. That is the content of a classical theorem of Loewner L]. (See also Velling $[\mathrm{V}]$.)

2. The length $\sigma$ of $f(C)$ is to be taken with multiplicity, if $f(C)$ is a multiple covering of the image.

3. Inequality (1) is sharp, with equality possible for each value of $\left|f^{\prime}(0)\right|$.

4. One can drop the condition (c) that $f(0)=0$. Analogous results hold for any value of $f(0)$. See Lemma 3 , the General Boundary Lemma, below.

5 . One does not need to assume that $f$ extends continuously to $b$. For example, if $f$ has a radial limit $c$ at $b$, with $|c|=1$, and if $f$ has a radial derivative at $b$, then that derivative also satisfies the inequality (11). More generally, if for some $b$ with $|b|=1$ there exists a sequence $z_{n}$ such that $z_{n} \rightarrow b$ and $f\left(z_{n}\right) \rightarrow c$ with $|c|=1$, then

$$
\varliminf_{z_{n} \rightarrow b}\left|\frac{f\left(z_{n}\right)-c}{\left|z_{n}\right|-|b|}\right| \geq \varliminf_{z_{n} \rightarrow b} \frac{1-\left|f\left(z_{n}\right)\right|}{1-\left|z_{n}\right|} \geq \frac{2}{1+\left|f^{\prime}(0)\right|} .
$$

Both Lemma 1 and the statement about radial limits are immediate consequences, since in either case we may choose $z_{n}=t_{n} b$ for $t_{n}$ real, $t_{n} \rightarrow 1$, and the left-hand side of (5) becomes $\left|f^{\prime}(b)\right|$.

Lemma 2 (Interior Schwarz Lemma). Let $f(z)$ satisfy conditions (a),(b),(c) of Lemma 1, Then

$$
|f(z)| \leq|z| \frac{|z|+\left|f^{\prime}(0)\right|}{1+\left|f^{\prime}(0)\right||z|} \text { for }|z|<1
$$


Proof. Let $g(z)=\frac{f(z)}{z}$. Then by the standard Schwarz Lemma, either $f$ is a rotation, or else $|g(z)|^{z}<1$ for $|z|<1$. In the former case, $\left|f^{\prime}(0)\right|=1$ and (6) holds trivially. So we need only consider the second case, where $|g(z)|<1$. Furthermore, since inequality (6) is unaffected by rotations, we may assume that $g(0)=f^{\prime}(0)=a$, where $0 \leq a<1$. Then (6) is equivalent to

$$
|g(z)| \leq \frac{|z|+a}{1+a|z|} \text { for }|z|<1, \text { with } a=g(0) .
$$

But that is an immediate consequence of the standard Schwarz-Pick version of the Schwarz Lemma, which says that $g$ must map each disk $|z|<r$ into the image of that disk under the linear fractional map

$$
G(z)=\frac{z+a}{1+a z}
$$

which is a circular disk whose diameter is the interval

$$
\left[\frac{a-r}{1-a r}, \frac{a+r}{1+a r}\right]
$$

of the real axis.

Hence,

$$
|z|=r \Rightarrow|g(z)| \leq \frac{a+r}{1+a r}=\frac{|z|+a}{1+a|z|},
$$

which proves (7), and hence (6).

Remarks 2. 1. For related sharpened forms of the interior Schwarz Lemma, see Mercer $[\mathrm{M}]$.

2. Inequality (7) is sharp, with equality for $g(z)=G(z), z=r$. Hence, inequality (6) is sharp, with equality for the function

$$
f(z)=z \frac{z+a}{1+a z}, 0 \leq a<1
$$

when $z$ is on the positive real axis. The same function gives equality in (11) when $b=1$.

3. When $f$ is not a rotation, (6) is a strict improvement on the standard Schwarz Lemma, since the second factor on the right is strictly less than 1 when $\left|f^{\prime}(0)\right|<1$.

Proof of Lemma 11. Let $f$ satisfy conditions (a), (b), (c) of Lemma 1. Then, using the upper bound (6) for $|f(z)|$, we have for any $b$ and $c$ with $|b|=1,|c|=1$,

$$
\left|\frac{f(z)-c}{|z|-|b|}\right| \geq \frac{1-|f(z)|}{1-|z|} \geq \frac{1+|z|}{1+\left|f^{\prime}(0)\right||z|} .
$$

As $|z| \rightarrow 1$, the right-hand side tends to $\frac{2}{1+\left|f^{\prime}(0)\right|}$.

This proves (5), and as noted in Remark 1.5 above, Lemma 1 follows.

Lemma 3 (The General Boundary Lemma). Under hypotheses (a), (b), and (d) of Lemma 1, one has

$$
\left|f^{\prime}(b)\right| \geq \frac{2}{1+\left|F^{\prime}(0)\right|} \frac{1-|f(0)|}{1+|f(0)|},
$$

where $F$ is defined in (9) below and satisfies $\left|F^{\prime}(0)\right| \leq 1$. 
Proof. Let

$$
F(z)=\frac{f(z)-f(0)}{1-\overline{f(0)} f(z)}
$$

Then $F$ satisfies the hypotheses of Lemma 1, and therefore

$$
\left|F^{\prime}(b)\right| \geq \frac{2}{1+\left|F^{\prime}(0)\right|}
$$

But a calculation gives

$$
F^{\prime}(z)=f^{\prime}(z) \frac{1-|f(0)|^{2}}{[1-\overline{f(0)} f(z)]^{2}}
$$

Since $|f(b)|=1$ implies

$$
|1-\overline{f(0)} f(b)| \geq 1-|\overline{f(0)} f(b)|=1-|f(0)|,
$$

we have

$$
\left|F^{\prime}(b)\right|=\left|f^{\prime}(b)\right| \frac{1-|f(0)|^{2}}{|1-\overline{f(0)} f(b)|^{2}} \leq\left|f^{\prime}(b)\right| \frac{1+|f(0)|}{1-|f(0)|} .
$$

Combining (10) and (11) yields (8).

Remarks 3 (Concluding Remarks). 1. An interesting special case of Lemma 1 is when $f^{\prime}(0)=0$, in which case inequality (II) implies $\left|f^{\prime}(b)\right| \geq 2$. Clearly equality holds for

$$
f(z)=e^{i \alpha} z^{2}, \quad \alpha \text { real. }
$$

Furthermore, that is the only case of equality; the same type of argument used to prove Lemmas 1 and 2yields a stronger inequality that implies $\left|f^{\prime}(b)\right|>2$ unless $f$ is of the form (12). More generally, the argument of the standard Schwarz Lemma shows that if $f(z)=\sum_{n=0}^{\infty} a_{n} z^{n}$ satisfies (a), (b) of Lemma 1 and if

$$
a_{0}=a_{1}=\cdots a_{k-1}=0
$$

then $\left|a_{k}\right| \leq 1$, and $\left|a_{k}\right|=1$ if and only if

$$
f(z)=e^{i \alpha} z^{k}, \quad \alpha \text { real. }
$$

Furthermore, either (14) holds, or else $|f(z)|<|z|^{k}$ for $|z|<1$. The argument of Lemma 2 yields the stronger result that

$$
|f(z)| \leq|z|^{k} \frac{|z|+\left|a_{k}\right|}{1+\left|a_{k}\right||z|} .
$$

Using (15) in the proof of Lemma 1 then shows that if also condition (d) of Lemma 1 holds, then

$$
\left|f^{\prime}(b)\right| \geq k+\frac{1-\left|a_{k}\right|}{1+\left|a_{k}\right|}
$$

It follows that $\left|f^{\prime}(b)\right| \geq k$, with equality only if $f$ is of the form (14).

2. A corollary of Lemma 3 is that under the same hypotheses, one has

$$
\left|f^{\prime}(b)\right| \geq \frac{1-|f(0)|}{1+|f(0)|}
$$

and the inequality is strict unless $f$ is an automorphism of the unit disk. In this context, see Carathéodory [C1], pp. 54-55, on Julia's Theorem, and [C2], pp. 25-27. 
3. For related results, and other types of boundary Schwarz Lemmas, see Carathéodory [C2], pp. 28-32, on the converse of Julia's Theorem, Pommerenke [P], p. 71, on the Julia-Wolff Lemma, and the paper of Burns and Krantz [BK]. See also [PS], paragraph 291 on p. 162 and p. 373, which gives the weaker inequality (2) together with the additional observation that if $b=f(b)=1$, then $f^{\prime}(1)$ is real and positive. For a different view of this last fact in the context of angular derivatives, see section 299 of Carathéodory [C2].

\section{REFERENCES}

[BK] Burns, D.M. and Krantz, S.G. "Rigidity of holomorphic mappings and a new Schwarz Lemma at the boundary." J. Amer. Math. Soc. 7 (1994) 661-676. MR 94j:32016

[C1] Carathéodory, C. Conformal Representation Cambridge University Press 1952. MR 13:734a

[C2] Carathéodory, C. Theory of Functions Vol. 2, New York, Chelsea 1954. MR 15:612b

[L] Löwner. K. "Untersuchungen über schlichte konforme Abbildungen des Einheitskreises. I." Math. Ann. 89 (1923) 103-121.

[M] Mercer, P.R. "Sharpened versions of the Schwarz Lemma." J. Math. Anal. Appl. 205 (1997) 508-511. MR 98e:30016

[O] Osserman, R. "A new variant of the Schwarz-Pick-Ahlfors Lemma." Manuscripta Mathematica 100 (1999) 123-124.

[OR] Osserman, R. and Ru, M. "An estimate for the Gauss curvature of minimal surfaces in $R^{m}$ whose Gauss map omits a set of hyperplanes." J. Differential Geometry 46 (1997) 578-593. MR 98k:53014

[PS] Pólya, G. and Szegö, G. Problems and Theorems of Analysis Vol. 2, New York, SpringerVerlag 1972. MR 57:5529

[P] Pommerenke, Ch. Boundary Behaviour of Conformal Maps, New York, Springer-Verlag 1992. MR 95b:30008

[V] Velling, J.A. "The uniformizations of rectangles, an exercise in Schwarz's Lemma." Amer. Math. Monthly 39 (1992) 112-115. MR 93b:30008

MSRI, 1000 Centennial Drive, Berkeley, California 94720-5070

E-mail address: osserman@msri.org 